\title{
EFFECTIVENESS STUDY OF CODE-AIDED AND NON- COdE-Aided ML-BASEd FeEdBACK PHASE SYNCHRONIZERS
}

\author{
N. Noels, H. Steendam and M. Moeneclaey \\ TELIN department, Ghent University, St-Pietersnieuwstraat 41, B-9000 GENT, BELGIUM \\ Tel: (+32) 926434 26, Fax: (+32) 92644295 \\ Mailto: \{nnoels, hs, mm\}@telin.UGent.be
}

\begin{abstract}
This paper investigates the effectiveness of a (noncode-aided ML-based FB phase synchronizer at the low operating signal-to-noise ratio of capacity-approaching codes. We show that the performance of the code-aided synchronizer is very close to that of a data-aided synchronizer that knows all data symbols in advance. This illustrates the optimality of the codeaided synchronizer. For the non-code-aided and the data-aided synchronizer, the linearized mean square phase error (MSPE) is evaluated analytically in the case of a first order loop. We demonstrate that, the MSPE of the non-code-aided synchronizer equals that of the data-aided synchronizer when the carrier phase is essentially constant and the loop filter gain is the same for both synchronizers, but that the non-code-aided synchronizer (as compared to the data-aided synchronizer) yields a larger MSPE due to phase fluctuations. This proves that codeaided FB phase estimation outperforms non-code-aided FB phase estimation when that the phase to be estimated is time-varying.
\end{abstract}

Carrier Synchronization, FB Phase Estimation, Errorcorrecting Codes

\section{INTRODUCTION}

The last decade has seen the development of powerful error correcting codes. The impressive bit error rate performance of the associated iterative decoding processes implicitly assumes coherent detection, meaning that the carrier phase must be recovered accurately before the data is decoded. However, since the decoder usually operates at extremely low signal-tonoise ratio (SNR), accurate carrier recovery is a challenging task. Numerous efforts to tackle this problem have resulted in a myriad of different receivers [1]-[9].

The iterative scheme in [4], which is based on the expectation-maximization algorithm, converges to the true maximum likelihood (ML) carrier phase estimate [10],[11]. Unfortunately, its performance rapidly degrades in the presence of a time-varying carrier phase. In [2], [5], [6], [7] and [9], feedback (FB) phase estimation has been adopted to cope with carrier phase variations. The ML-based receiver in [9] combines the low complexity from the approach in [4]

This work has been supported by the Interuniversity Attraction Poles Program - Belgian State - Federal Office for Scientific, Technical and Cultural Affairs with the ability to automatically track a slowly varying carrier phase. Simulation results in [9] show the interesting potential of this approach. As opposed to the algorithms in [2], [5] and [6], the derivation of the phase estimation algorithm stems directly from the ML criterion. Moreover, its computational complexity is lower than that of the algorithms in [7] and [8], which modify the decoder operation by either taking into account the phase statistics or using per-survivor phase estimates inside the decoder.

This contribution zooms in on the approach that was adopted in [9]. By means of theoretical analysis and computer simulations we compare the tracking performances resulting from the iterative code-aided (CA) synchronizer from [9], the data-aided (DA) synchronizer which knows all transmitted symbols in advance, and the non-code-aided (NCA) synchronizer which neglects the underlying encoding rule. We illustrate the optimality of [9], and show that CA FB phase estimation is more effective than NCA FB phase estimation when the phase to be estimated is time-varying; when the carrier phase is constant over the observation interval, both synchronizers yield essentially the same mean square phase error (MSPE).

\section{BRIEF OVERVIEW OF ESTIMATION THEORY}

Suppose that one is able to produce from an observation vector $\mathbf{r}=\left\{r_{k}\right\}_{k=0}^{K-1}$ an unbiased estimate $\hat{\theta}$ of an unknown but deterministic parameter $\theta$, i.e., $E_{\mathrm{r}}[\hat{\theta}]=\theta$ for all $\theta$. Then the MSPE is lower bounded by the Cramer-Rao Bound (CRB) [12]: $E_{\mathbf{r}}\left[(\hat{\theta}-\theta)^{2}\right] \geq \mathrm{CRB}$, where

$$
C R B=E_{\mathbf{r}}\left[\left(\left.\frac{d \ln (p(\mathbf{r} ; \tilde{\theta}))}{d \widetilde{\theta}}\right|_{\widetilde{\theta}=\theta}\right)^{2}\right]^{-1}=E_{\mathbf{r}}\left[\left(L^{\prime}(\theta)\right)^{2}\right]^{-1}
$$

In (1), $p(\mathbf{r} ; \tilde{\theta})$ denotes the probability density function of $\mathbf{r}$, corresponding to a given trial value $\widetilde{\theta}$ of $\theta ; p(\mathbf{r} ; \widetilde{\theta})$ is called the likelihood function of $\tilde{\theta}$, while $\ln (p(\mathbf{r} ; \tilde{\theta}))$ is the $\log$ likelihood function of $\tilde{\theta}$. The expectation $\mathrm{E}_{\mathrm{r}}[$.$] in (1) is with$ 
respect to $\mathrm{p}(\mathrm{r} ; \theta), \mathrm{L}(\widetilde{\theta})$ is a shorthand notation for $\ln (p(\mathbf{r} ; \widetilde{\theta}))$ and $\mathrm{L}^{\prime}(\theta)$ denotes the derivative of $\mathrm{L}(\tilde{\theta})$ with respect to evaluated at $\tilde{\theta}=\theta$.

Now consider the transmission of an arbitrary sequence of complex-valued symbols $\mathbf{a}=\left\{a_{k}\right\}_{k=0}^{K-1}$ over an additive white Gaussian noise (AWGN) channel. The joint probability mass function of the symbols $\left\{a_{k}\right\}_{k=0}^{K-1}$ is denoted as p(a). Assuming linear modulation using square-root Nyquist transmit pulses, and matched filtering at the correct decision instants, the discrete-time baseband observation is given by

$$
\mathrm{r}_{\mathrm{k}}=\mathrm{a}_{\mathrm{k}} \exp (\mathrm{j} \theta)+\mathrm{w}_{\mathrm{k}}, \mathrm{k}=0, \ldots, \mathrm{K}-1
$$

where $\theta$ is the unknown but deterministic carrier phase. The sequence $\left\{w_{k}\right\}_{k=0}^{K-1}$ consists of independent zero-mean complex-valued Gaussian noise terms; $\operatorname{Re}\left[\mathrm{w}_{\mathrm{k}}\right]$ and $\operatorname{Im}\left[\mathrm{w}_{\mathrm{k}}\right]$ are statistically independent, and have a variance equal to $\mathrm{N}_{0} / 2$.

The derivative of the $\log$-likelihood function $\ln (p(\mathbf{r} ; \tilde{\theta}))$ resulting from (2) with respect to $\tilde{\theta}$ can be manipulated into the following form [13]:

$$
L^{\prime}(\widetilde{\theta})=\frac{d \ln (p(\mathbf{r} ; \widetilde{\theta}))}{d \widetilde{\theta}}=\frac{2}{N_{0}} \sum_{k=0}^{K-1} \operatorname{Im}\left[A_{k}^{*}(\mathbf{r}, \widetilde{\theta}) r_{k} e^{-j \widetilde{\theta}}\right]
$$

where

$$
A_{k}(\mathbf{r}, \tilde{\theta})=E\left[a_{k} \mid \mathbf{r} ; \tilde{\theta}\right]=\sum_{m=0}^{M-1} \operatorname{Pr}\left[a_{k}=s_{m} \mid \mathbf{r} ; \tilde{\theta}\right] s_{m}
$$

is the a posteriori expectation of the symbol $\mathrm{a}_{\mathrm{k}}$ conditioned on $\mathbf{r}$ and $\theta$, with $\operatorname{Pr}\left[a_{k}=s_{m} \mid \mathbf{r} ; \tilde{\theta}\right]$ denoting the marginal a posteriori probability (APP) of the symbol $\mathrm{a}_{\mathrm{k}}$, and $\left\{s_{m}\right\}_{m=0}^{M-1}$ the set of constellation points with symbol energy $\mathrm{E}_{\mathrm{s}}=$ $\frac{1}{M} \sum_{m=0}^{M-1}\left|s_{m}\right|^{2}$. Computation of the CRB requires the substitution of (3) into (1), and the evaluation of the expectation in (1).

When the data symbol vector a consists of known pilot symbols $\left\{a_{p, k}\right\}_{k=0}^{K-1}$, we obtain $\operatorname{Pr}\left[a_{k}=s_{m} \mid \mathbf{r} ; \tilde{\theta}\right]$ equal to 1 for $s_{m}=a_{p, k}$ and zero otherwise, yielding $A_{k}(\mathbf{r}, \widetilde{\theta})=\mathrm{a}_{\mathrm{p}, \mathrm{k}}$ in (4). The log-likelihood function and the CRB that correspond to the transmission of pilot symbols are denoted $L_{p s}($.$) and$ $\mathrm{CRB}_{\mathrm{ps}}$, respectively. In the case of an MPSK constellation or when the marginal distribution $\mathrm{p}\left(\mathrm{a}_{\mathrm{k}}\right)$ is uniform over the signal constellation $^{1}$, it is easily verified from (3) and (1) that that $\mathrm{CRB}_{\mathrm{ps}}=\mathrm{N}_{0} /\left(2 \mathrm{KE}_{\mathrm{s}}\right)$, (irrespective of the details of the code).

\footnotetext{
${ }^{1}$ This property holds for the large majority of codes of practical interest.
}

In the case of uncoded transmission, the symbols $\left\{a_{m}\right\}_{k=0}^{K-1}$ are statistically independent, so the APPs of $\mathrm{a}_{\mathrm{k}}$ reduce to:

$$
\operatorname{Pr}\left[a_{k}=s_{m} \mid \mathbf{r} ; \tilde{\theta}\right]=\operatorname{Pr}\left[a_{k}=s_{m} \mid r_{k} ; \tilde{\theta}\right]=\frac{p\left(r_{k} \mid a_{k}=s_{m} ; \tilde{\theta}\right)}{\sum_{i=0}^{M-1} p\left(r_{k} \mid a_{k}=s_{i} ; \tilde{\theta}\right)}
$$

where

$$
p\left(r_{k} \mid a_{k}=s_{m} ; \tilde{\theta}\right)=\exp \left(\frac{-1}{N_{0}}\left|r_{k}-s_{m} e^{j \widetilde{\theta}}\right|^{2}\right)
$$

As (6) depends only on $r_{k}$, we will denote the corresponding a posteriori average of the symbol $\mathrm{a}_{\mathrm{k}}$ as $A_{k}\left(r_{k} ; \widetilde{\theta}\right)$. The loglikelihood function and the CRB that correspond to the transmission of statistically independent symbols $\left\{a_{k}\right\}_{k=0}^{K-1}$ are denoted $\mathrm{L}_{\text {ind }}($.$) and \mathrm{CRB}_{\text {ind }}$, respectively.

This paper considers systems with an iteratively decodable error-control code (turbo-, LDPC-codes). In this case, the APPs in (4) are a function of all components of the vector $\mathbf{r}$. To avoid the computational complexity associated with their exact evaluation, the marginal APPs are approximately computed by means of the iterative application of the sumproduct (SP) algorithm on a factor graph with cycles [13],[14].

\section{ML-BASED FB PHASE ESTIMATION}

In a first order FB phase estimator or phase-locked loop (PLL), the phase estimate is updated once per symbol interval, according to the following recursion [15]

$$
\hat{\theta}_{k+1}=\hat{\theta}_{k}+\lambda x_{k}
$$

In (7), $x_{k}$ denotes the phase error detector (PED) output. The recursion starts with an initial phase estimate $\hat{\theta}_{0}$, that can be obtained from a feedforward synchronizer operating on a short pilot sequence.

In the following, we consider three types of ML-based PEDs. The DA PED (based on $L_{p s}^{\prime}(\tilde{\theta})$ ) assumes that all data symbols are known. The NCA PED (based on $L_{\text {ind }}^{\prime}(\tilde{\theta})$ ) assumes that the data symbols are independent, whereas the CA PED (based on the true $L^{\prime}(\tilde{\theta})$ ) takes the code properties into account. We obtain from (3) that

$$
x_{k}=\frac{1}{E_{s}} \begin{cases}\operatorname{Im}\left[a_{k}^{*} r_{k} e^{-j \hat{\theta}_{k}}\right], & \text { DA operation } \\ \operatorname{Im}\left[A_{k}^{*}\left(r_{k}, \hat{\theta}_{k}\right) r_{k} e^{-j \hat{\theta}_{k}}\right], & \text { NCA operation } \\ \operatorname{Im}\left[A_{k}^{*}\left(\mathbf{r}, \hat{\theta}_{k}\right) r_{k} e^{-j \hat{\theta}_{k}}\right], & \text { CA operation }\end{cases}
$$

Comparison of the PED outputs for NCA and CA operation with that for DA operation indicates that the a posteriori mean $\mathrm{A}_{\mathrm{k}}$ can be considered as a soft decision (SD) regarding $\mathrm{a}_{\mathrm{k}}$, based upon the received sample $\mathrm{r}_{\mathrm{k}}$ and the phase estimate $\hat{\theta}_{k}$. 
Note from (8) that the DA and NCA PED output depend only on $\left(r_{k}, \hat{\theta}_{k}\right)$; this is in contrast with the CA PED output whose computation depends on the entire vector $\mathbf{r}$ : all $\mathrm{K}$ samples $\left\{r_{k}\right\}_{k=0}^{K-1}$ have to be rotated over an angle $-\hat{\theta}_{k}$, and fed to the $\mathrm{SP}$ algorithm for producing the SD $A_{k}\left(\mathbf{r}, \hat{\theta}_{k}\right)$. Hence, in the case of $\mathrm{CA}$ operation, the entire received block must be processed $\mathrm{K}$ times, whereas the received block is processed only once in the case of DA or NCA operation.

In order to avoid the high computational complexity resulting from the CA PED, the following iterative CA PLL has been proposed in [9]. During the i-th iteration, the FB synchronizer generates estimates $\left\{\hat{\theta}_{k}^{(i)}\right\}_{k=0}^{K-1}$ essentially according to (7), but with the PED output given by

$$
x_{k}=\frac{1}{E_{s}} \operatorname{Im}\left[A_{k}^{*}\left(\mathbf{r}, \hat{\theta}^{(i-1)}\right) e^{-j \hat{\theta}_{k}^{(i)}}\right], \quad \text { iterative CA operation }
$$

where $\hat{\boldsymbol{\theta}}^{(i-1)}=\left\{\hat{\theta}_{k}^{(i)}\right\}_{k=0}^{K-1}$, and $A_{k}\left(\mathbf{r}, \hat{\theta}^{(i-1)}\right)$ is the a posteriori expectation of the symbol $a_{\mathrm{k}}$ conditioned on $\mathbf{r}$ and $\hat{\theta}^{(i-1)}$. Hence, from the phase vector $\hat{\boldsymbol{\theta}}^{(i-1)}$, the received vector $\mathbf{r}$ is processed to compute $\left\{A_{k}\left(\mathbf{r}, \hat{\theta}^{(i-1)}\right)\right\}_{k=0}^{K-1}$, after which the PLL generates the phase vector $\hat{\theta}^{(i)}$. The iterative process is initialized by means of a phase vector $\hat{\theta}^{(0)}$, that can be obtained from a PLL with NCA operation. When convergence is achieved after $\mathrm{n}$ iterations, the vector $\mathbf{r}=\left\{r_{k}\right\}_{k=0}^{K-1}$ has been processed $\mathrm{n}$ times. When $\mathrm{n}<<\mathrm{K}$, considerable savings in computation time have been obtained as compared to the noniterative PLL that uses the CA PED output from (8).

Fig. 1 shows the receiver structure for a parallel concatenated turbo code. The operation mode (DA, NCA or CA) of the PLL is determined by the position of the switch.

\section{Tracking Performance Analysis}

In order to allow a time varying carrier phase, the observation model (2) is modified into

$$
\mathrm{r}_{\mathrm{k}}=\mathrm{a}_{\mathrm{k}} \exp \left(\mathrm{j} \theta_{\mathrm{k}}\right)+\mathrm{w}_{\mathrm{k}}, \quad \mathrm{k}=0, \ldots, \mathrm{K}-1
$$

where $\theta_{\mathrm{k}}$ is the phase during the $\mathrm{k}$-th symbol interval. The carrier phase is assumed to perform a random walk characterized by i.i.d. Gaussian increments $\Delta_{\mathrm{k}}$ with zero mean and standard deviation $\sigma_{\Delta}$. It is assumed that $\left\{\mathrm{w}_{\mathrm{k}}\right\}$ and $\left\{\Delta_{\mathrm{k}}\right\}$ are statistically independent, and that $\theta_{0}$ is uniformly distributed in $[-\pi, \pi]$.

We first consider the performance of the DA and the NCA phase estimator. We define the phase estimation error during the k-th symbol period as $\phi_{k}=\theta-\hat{\theta}_{k}$. The DA and NCA PED outputs from (8) can be decomposed as the sum of their average $S\left(\phi_{k}\right)$ and their zero-mean statistical fluctuation $N_{k}\left(\phi_{k}\right)$, with

$$
S\left(\phi_{k}\right)=E\left[x_{k}\right] \quad N_{k}\left(\phi_{k}\right)=x_{k}-E\left[x_{k}\right]
$$

denoting the PED characteristic and the loop noise of the synchronizer. We show in the Appendix that $\mathrm{S}(0)=0$. Assuming small phase errors, the following linearization applies:

$$
x_{k}=S^{\prime}(0) \phi_{k}+N_{k}(0)
$$

where $\mathrm{S}^{\prime}(0)$ is the slope of the PED characteristic and $\mathrm{N}_{\mathrm{k}}(0)$ is the loop noise at $\phi_{\mathrm{k}}=0$. We show in the Appendix that $\mathrm{E}\left[\mathrm{N}_{\mathrm{k}}(0) \mathrm{N}_{\mathrm{k}},(0)\right]=\mathrm{S}^{\prime}(0) \mathrm{N}_{0} /\left(2 \mathrm{E}_{\mathrm{s}}\right) \delta_{\mathrm{k}-\mathrm{k}^{\prime}}$, where

$$
S^{\prime}(0)= \begin{cases}1, & \text { DA operation } \\ \left(R_{\text {ind }}\right)^{-1}=C R B_{p s} / C R B_{\text {ind }}, & \text { NCA operation }\end{cases}
$$

Substituting (12) into (7) we obtain the model shown in Fig. 2, where $H(z)=\lambda S^{\prime}(0) /\left(z-1+\lambda S^{\prime}(0)\right)$ is the closed-loop response (in the z-domain) of the synchronizer. It is evident from Fig. 2 that the MSPE $E\left[\left(\phi_{\mathrm{k}}\right)^{2}\right]$ does not depend on $\mathrm{k}$, and consist of two contributions that are caused by the AWGN and the phase noise $(\mathrm{PN})$, respectively. We obtain $\mathrm{E}\left[\left(\phi_{\mathrm{k}}\right)^{2}\right]=\mathrm{MSPE}_{\mathrm{AWGN}}+$ $\mathrm{MSPE}_{\mathrm{PN}}$, with

$$
\begin{aligned}
& \operatorname{MSPE}_{A W G N}=E\left[\left(N_{k}(0)\right)^{2}\right] \frac{2 B_{L} T}{S^{\prime}(0)^{2}}=\frac{N_{0}}{E_{s}} \frac{B_{L} T}{S^{\prime}(0)} \\
& \operatorname{MSPE}_{P N}=E\left[\left(\Delta_{k}\right)^{2}\right] \frac{2 B_{L} T}{\left(\lambda S^{\prime}(0)\right)^{2}} \cong \sigma_{\Delta}^{2} \frac{2 B_{L} T}{\left(\lambda S^{\prime}(0)\right)^{2}}
\end{aligned}
$$

where $B_{L} T$ is the one-side bandwidth (normalized to the symbol rate) of $\mathrm{H}(\mathrm{z})$. We have

$$
B_{L} T=T \int_{0}^{1 / 2 T}\left|H\left(e^{j 2 \pi F T}\right)\right|^{2} d f=\frac{\lambda S^{\prime}(0)}{2\left(2-\lambda S^{\prime}(0)\right)} \cong \frac{\lambda S^{\prime}(0)}{4}
$$

where the approximation is valid for small $\lambda$. Substituting (15) into (14) learns that a larger value of $\lambda$ yields a larger AWGN contribution but a smaller PN contribution, and vice versa. When the carrier phase is time-varying $\left(\sigma_{\Delta} \neq 0\right)$, there exists an optimal value for $\lambda$ that minimizes the MSPE. When the carrier phase is time-invariant $\left(\sigma_{\Delta}=0\right)$, the MSPE can be made arbitrarily small by reducing the value of $\lambda$. A small $\lambda$, however, implies a large acquisition time $\mathrm{T}_{\text {acq }}$ (i.e. time for the MSPE to evolve from its initial value to the steady state value of (14)). Assuming a uniformly distributed initial phase error, the mean time to acquire in the absence of noise is well approximated by $\frac{E\left[T_{a c q}\right]}{T}=\frac{1}{2 B_{L} T} \cong \frac{2}{\lambda S^{\prime}(0)}[15]$. 


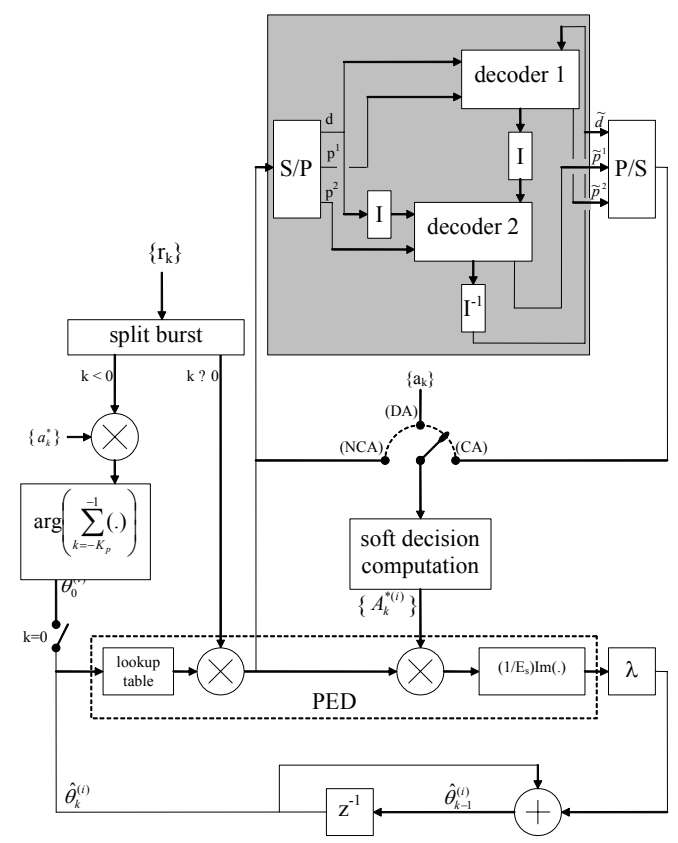

Fig. 1: Turbo receiver with $M L$-based PLL. Legend: $I\left(T^{1}\right)=$ (de)interleaver, $S / P(P / S)=$ serial-to-parallel (de)converter, $d(\tilde{d})=$ observation (marginal APPS) of information bit, $p_{i}\left(\widetilde{p}_{i}\right)=$ observation (marginal APPs) of parity bit from encoder $i \in\{1,2\}$.

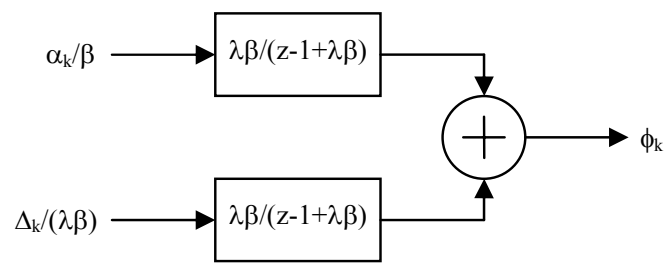

Figure 2: Contributions to the phase error $\phi_{k}$

Computing the exact tracking performance of the iterative CA FB phase estimator is much more difficult than for NCA and DA synchronizers, because of the iterations involved and the dependence of the soft decisions on the entire phase vector. Instead we will proceed assuming that, at the normal operating SNR of the considered code, the MSPE resulting from the iterative CA phase estimator converges to the MSPE resulting from a fictitious DA phase estimator that knows all data symbols in advance; the latter MSPE is given by (14) with $\mathrm{S}^{\prime}(0)=1$.

A motivation for this assumption reads as follows. Note that in (8) the CA PED output reduces to the DA PED output when the APP $\operatorname{Pr}\left[s_{m} \mid \mathbf{r} ; \hat{\theta}_{k}\right]$ is one for $\mathrm{s}_{\mathrm{m}}=\mathrm{a}_{\mathrm{k}}$ and zero otherwise. This indicates that after convergence, the CA PLL essentially behaves like the DA PLL, provided that the ratios $\mathrm{R}\left(\mathrm{s}_{\mathrm{m}}, \mathrm{a}_{\mathrm{k}} \mid \mathbf{r}\right.$;
$\left.\hat{\theta}_{k}\right)=\operatorname{Pr}\left[s_{m} \mid \mathbf{r} ; \hat{\theta}_{k}\right] / \operatorname{Pr}\left[a_{k} \mid \mathbf{r} ; \hat{\theta}_{k}\right]$ are likely to be much smaller than 1 for all $s_{m} \neq a_{k}$ and all $\mathrm{k}=0, \ldots, \mathrm{K}-1$. Let us introduce the indicator function $I_{y}(k)$, which equals one when $R\left(s_{m}, a_{k} \mid r\right.$; $\hat{\theta}_{k}$ ) $\geq \mathrm{y}$ for at least one $\mathrm{s}_{\mathrm{m}} \neq \mathrm{a}_{\mathrm{k}}$, and equals zero otherwise. Then we obtain

$E\left[\frac{1}{K} \sum_{k=0}^{K-1} I_{y}(k)\right]=\frac{1}{M^{\rho K}} \sum_{\mathbf{a} \in \xi}\left(\sum_{k=0}^{K-1} \operatorname{Pr}\left[\bigcup_{s_{m} \neq a_{k}} R\left(s_{m}, a_{k} \mid \mathbf{r} ; \hat{\theta}_{k}\right) \geq y\right]\right)(16)$

where $\xi$ denotes the set of legitimate coded symbol sequences of length $\mathrm{K}$. We assume that $\operatorname{Pr}[\mathbf{a}]=\mathrm{M}^{-\mathrm{\rho K}}$ for $\mathbf{a} \in \xi$ and $\operatorname{Pr}[\mathbf{a}]$ $=0$ otherwise, where the quantities $\rho$ and $M$ denote the rate of the code and the number of constellation points, respectively. With $\mathrm{y}=1$ and $\hat{\theta}_{k}=\theta$ for all $\mathrm{k}$, equation (16) is nothing but the (very small) symbol error rate resulting from an optimal maximum a posteriori probability symbol decoder [16]. Hence, for small phase errors, the fraction of symbol intervals for which $\mathrm{I}_{\mathrm{y}}(\mathrm{k})=1$ is very small, so that we can safely assume that the CA PLL operation closely resembles the DA PLL operation, at the normal operating SNR of the code.

\section{NCA VERSUS DA FB PHASE ESTIMATION}

In this section we compare the operation of the NCA FB phase estimator with that of the DA FB phase estimator; we recall that in the previous section we have pointed out that the latter estimator essentially behaves like the converged CA estimator. At the low SNR supported by capacity-approaching codes it is not possible to compute reliable data decisions without taking into account the code structure; hence, we expect the NCA PLL to perform significantly worse than a DA PLL with perfect knowledge on the data symbols. Using superscripts ' $N C A$ ' and 'DA' to denote the operation mode of the PLL, and assuming that $\lambda^{\mathrm{NCA}}=\lambda^{\mathrm{DA}}$, we obtain from the previous section:

$$
\frac{E\left[T_{a c q}^{N C A}\right]}{E\left[T_{a c q}^{D A}\right]}=R_{\text {ind }} \quad \frac{M S E_{A W G N}^{N C A}}{M S E_{A W G N}^{D A}}=1 \quad \frac{M S E_{P N}^{N C A}}{M S E_{P N}^{D A}}=R_{\text {ind }}
$$

At the normal operating $\mathrm{SNR}$ of powerful codes, $\mathrm{R}_{\text {ind }}$ is significantly larger than one [13]; hence, the acquisition time and the PN contribution to the MSPE are larger for NCA operation than for DA operation. This meets our expectations. The AWGN contribution to the MSPE, however, is the same for NCA and DA operation. Hence, when the iterative CA PLL is initialized by means of a NCA recursion, the MSPE will not further reduce when performing iterations in the CA mode. This surprising result can be explained as follows (see Table 1 and the first equation in (14)). For given values of $\lambda$ and $E_{S} / N_{0}$, the reduction of the PED slope $S^{\prime}(0)$ of the NCA PLL (as compared with the DA PLL) is precisely compensated by the reduction of the loop bandwidth $2 \mathrm{~B}_{\mathrm{L}} \mathrm{T}$ and of the phase noise variance $\mathrm{E}\left[\left(\alpha_{\mathrm{k}}\right)^{2}\right]$. 
Table1: Computation of the AWGN contribution to the MSPE of a FB estimator

\begin{tabular}{l|ll}
\hline & DA & NCA \\
\hline $\mathrm{S}^{\prime}(0)$ & 1 & $1 / \mathrm{R}_{\text {ind }}$ \\
$\mathrm{E}\left[\left(\mathrm{N}_{\mathrm{k}}(0)\right)^{2}\right]$ & $\mathrm{N}_{0} /\left(2 \mathrm{E}_{\mathrm{s}}\right)$ & $\mathrm{N}_{0} /\left(2 \mathrm{E}_{\mathrm{s}} \mathrm{R}_{\text {ind }}\right)$ \\
$2 \mathrm{~B}_{\mathrm{L}} \mathrm{T}$ & $\lambda / 2$ & $\lambda /\left(2 \mathrm{R}_{\text {ind }}\right)$ \\
\hline $\mathrm{MSPE}=\mathrm{E}\left[\left(\mathrm{N}_{\mathrm{k}}(0)\right)^{2}\right]\left(2 \mathrm{~B}_{\mathrm{L}} \mathrm{T}\right)\left(1 / \mathrm{S}^{\prime}(0)\right)^{2}$ & $\lambda \mathrm{N}_{0} /\left(4 \mathrm{E}_{\mathrm{s}}\right)$ & $\lambda \mathrm{N}_{0} /\left(4 \mathrm{E}_{\mathrm{s}}\right)$ \\
\hline
\end{tabular}

Let us consider a rate $1 / 3$ turbo-coded BPSK signal. The turbo encoder consists of the parallel concatenation of two identical non-recursive systematic convolutional encoders with generator polynomials $(21)_{8}$ and $(37)_{8}$ in octal notation, separated by a pseudo-random interleaver of size 333 bits. The signal is transmitted over an AWGN channel affected by a random walk PN process (see (10)), and applied to the receiver of Figure 1, with $\lambda=0.04$. In Figs. 3 and 4 we have plot the MSPE at the PLL output as a function of the symbol index $\mathrm{k}$. Results are shown for DA, NCA and iterative CA operation; note that the iteration $\mathrm{i}=0$ of the CA PLL is a NCA recursion. A sequence 16 pilot symbols that precedes the actual data symbols is used to produce $\hat{\theta}_{0}$. The ratio $\mathrm{E}_{\mathrm{s}} / \mathrm{N}_{0}$ is set to $-2.77 \mathrm{~dB}$, which has been verified (outside the scope in

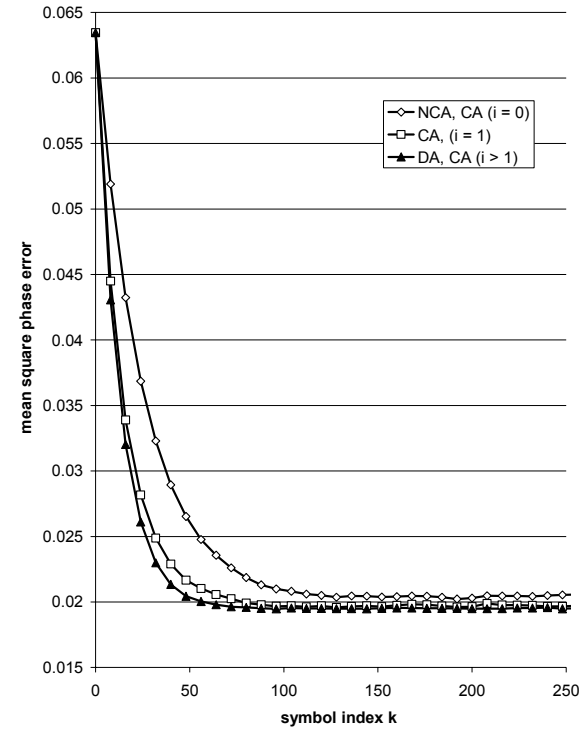

Figure 3: MSPE of a first order PLL with $\lambda=0.04$, tracking a constant carrier phase

\section{CONCLUSIONS}

This contribution has studied the effectiveness of CA and NCA ML-based PLLs at the low operating SNR of iteratively decodable codes. The NCA synchronizer assumes that the data symbols are independent, whereas the CA synchronizer takes the code properties into account. The performance of a fictitious DA synchronizer that know all data symbols in advance served as a reference to which the performance of the NCA synchronizers and the CA synchronizers was compared. this paper) to be in the operating range of the code. The carrier phase is assumed to be either constant over the observation interval (Fig. 3), or to perform a random walk with $\sigma_{\Delta}=2^{\circ}$ (Fig. 4). We make the following observations

- When the number of iterations grows, the MSPE resulting from the CA synchronizer converges to the DA synchronizer MSPE.

- The NCA synchronizer yields practically the same steady state MSPE as the DA synchronizer, when the carrier phase is constant.

- The NCA synchronizer yields a larger steady state MSPE than the DA synchronizer, when the carrier phase performs a random walk.

- The DA synchronizer acquires faster than the NCA synchronizer.

The first observation confirms the validity of the assumption made at the end of Section 4. The other observations are consistent with (17) and with the value of $R_{\text {ind }}$ at $E_{s} / N_{0}=-2.77$ $\mathrm{dB}$ (has been verified outside the scope of this paper).

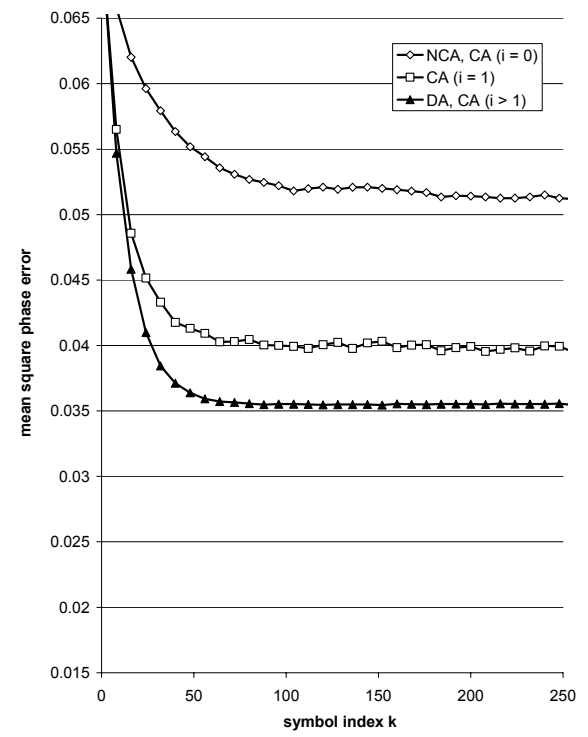

Figure 4: MSPE of a first order PLL with $\lambda=0.04$, tracking Wiener phase noise with $\sigma_{\Delta}=2$ degrees

We have motivated (both analytically and by simulations) that, under normal operating conditions, the MSPE resulting from the CA synchronizer converges to that of the DA synchronizer. This illustrates the optimality of the CA MLbased FB phase estimator.

We have demonstrated that the linearized MSPE of the NCA FB synchronizer equals that of the DA FB synchronizer, when the carrier phase is essentially constant over the observation interval and the loop filter gain is the same for both synchronizers. This surprising result implies that CA FB 
phase estimation is ineffective when the phase to be estimated is time-invariant.

We have also shown that the presence of a random walk phase noise process results in a NCA feeback phase synchronizer MSPE that is $R_{\text {ind }}$ times larger than the DA FB synchronizer MSPE. Here, $\mathrm{R}_{\text {ind }}=\mathrm{CRB}_{\text {ind }} / \mathrm{CRB}_{\mathrm{ps}}$ with $\mathrm{CRB}_{\text {ind }}$ and $\mathrm{CRB}_{\mathrm{ps}}$ denoting the Cramer-Rao lower bound on the MSPE of any unbiased estimator operating on the noisy observation of a signal with uncoded symbols and pilot symbols, respectively. At the normal operating SNR of powerful codes, $\mathrm{CRB}_{\text {ind }} / \mathrm{CRB}_{\mathrm{ps}}$ is significantly larger than 1 , which illustrates the effectiveness of CA FB phase estimation in the presence of phase noise.

\section{APPENDIX}

\section{$\mathrm{CRB}_{\text {ps }}$ and $\mathrm{CRB}_{\text {ind }}$}

We first show that, in the case of an MPSK constellation or when the marginal a priori distribution $p\left(a_{k}\right)$ is uniform over the signal constellation,

$$
\mathrm{CRB}_{\mathrm{ps}}=\mathrm{N}_{0} /\left(2 \mathrm{KE}_{\mathrm{s}}\right) \text {, }
$$

irrespective of the details of the code. Replacing in (5) the a posteriori averages $A_{k}(\mathbf{r}, \theta)$ with the true data symbols $\mathrm{a}_{\mathrm{k}}$, we obtain from (1)

$$
C R B_{p s}=\left(\frac{2}{N_{0}} \sum_{k=0}^{K-1}\left|a_{k}\right|^{2}\right)^{-1}
$$

In the case of an M-PSK constellation, $\left|\mathrm{a}_{\mathrm{k}}\right|^{2}=1$ for all $\mathrm{k}$; hence, (A1) follows directly from (A2). In the case of a long sequence in which the mutual dependency between the individual symbols is relatively weak (common situation for turbo and LDPC codes), the statistical fluctuation of $\sum_{k=0}^{K-1}\left|a_{k}\right|^{2}$ in (A2) can be ignored as compared to its mean. When the marginal a priori distribution $\mathrm{p}\left(\mathrm{a}_{\mathrm{k}}\right)$ is uniform ${ }^{2}$ over the constellation, this also yields (A1).

Further, it is easily verified from (1) and (5) with the APPs in (6) replaced with (7)-(8) that

$$
\begin{aligned}
C R B_{\text {ind }} & =\left(\sum_{k=0}^{K-1} E\left[\left(\frac{d \ln \left(p\left(r_{k} ; \theta\right)\right)}{d \theta}\right)^{2}\right]\right)^{-1} \\
& =\frac{1}{K}\left(E\left[\left(\frac{d \ln \left(p\left(r_{k} ; \theta\right)\right)}{d \theta}\right)^{2}\right]\right)^{-1}
\end{aligned}
$$

\footnotetext{
${ }^{2}$ This property holds for the large majority of codes of practical interest.
}

where the probability density $p\left(r_{k} ; \widetilde{\theta}\right)$ is given by $\sum_{i=0}^{M-1} p\left(r_{k} \mid a_{k}=s_{i} ; \tilde{\theta}\right) p\left(a_{k}=s_{i}\right) \quad$ with $p\left(r_{k} \mid a_{k}=s_{i} ; \tilde{\theta}\right)$ as in (8).

\section{Lineair performance analysis of DA and NCA PLL}

We first consider NCA feedback phase synchronization. Taking in equation (5) $\mathbf{r}=\mathrm{r}_{\mathrm{k}}$ and $\widetilde{\theta}=\hat{\theta}_{k}$, we find that the NCA PED output (13) can be rewritten as follows

$$
x_{k}=\frac{1}{E_{s}} \operatorname{Im}\left[A_{k}^{*}\left(r_{k}, \hat{\theta}_{k}\right) r_{k} e^{-j \hat{\theta}_{k}}\right]=\frac{N_{0}}{2 E_{s}} \frac{d \ln \left(p\left(r_{k} ; \hat{\theta}_{k}\right)\right)}{d \hat{\theta}_{k}}
$$

Taking the first and the second derivative (with respect to $\theta$ ) of both sides of the normalization constraint $\int p\left(r_{k} ; \theta\right) d r_{k}=1$, and using $d F(x) / d x=F(x)\{d \ln (F(x)) / d x\}$ it can be verified that

$$
\begin{aligned}
& E\left[\frac{d \ln \left(p\left(r_{k} ; \theta\right)\right)}{d \theta}\right]=0 \\
& E\left[\left(\frac{d \ln \left(p\left(r_{k} ; \theta\right)\right)}{d \theta}\right)^{2}\right]=-E\left[\frac{d^{2} \ln \left(p\left(r_{k} ; \theta\right)\right)}{d \theta^{2}}\right]
\end{aligned}
$$

It follows directly from $\phi_{k}=\theta-\hat{\theta}_{k}$ and (A7) that the (A4) equals zero at $\phi=0$. Taking into account (10) and $\phi_{k}=\theta-\hat{\theta}_{k}$, the PED slope $\mathrm{S}^{\prime}(0)$ and loop noise $\mathrm{N}_{\mathrm{k}}(0)$ at $\phi_{\mathrm{k}}=0$, resulting from (A4) are given by

$$
\begin{aligned}
& S^{\prime}(0)=-\frac{N_{0}}{2 E_{s}} E\left[\frac{d^{2} \ln \left(p\left(r_{k} ; \theta\right)\right)}{d \theta^{2}}\right] \\
& N_{k}(0)=\frac{N_{0}}{2 E_{s}} \frac{d \ln \left(p\left(r_{k} ; \theta\right)\right)}{d \theta}
\end{aligned}
$$

Because of the statistical properties of $\left\{r_{\mathrm{k}}\right\}$, the loop noise at $\phi_{\mathrm{k}}=0$ is white $^{3}$ and its power spectral density is given by

$$
E\left[\left(N_{k}(0)\right)^{2}\right]=\left(\frac{N_{0}}{2 E_{s}}\right)^{2} E\left[\left(\frac{d \ln \left(p\left(r_{k} ; \theta\right)\right)}{d \theta}\right)^{2}\right]
$$

Taking into account (A1) and (A3), we obtain

$$
S^{\prime}(0)=\frac{C R B_{p s}}{C R B_{\text {ind }}} \quad E\left[\left(N_{k}(0)\right)^{2}\right]=\frac{N_{0}}{2 E_{s}} \frac{C R B_{p s}}{C R B_{\text {ind }}}
$$

\footnotetext{
${ }^{3}$ In the case of uncoded transmission this follows directly from the fact that $r_{k}$ and $r_{k}$, are independent for $k \neq k$, but it can be shown analytically (outside the scope of this paper) that this property holds independently of the code properties.
} 
It is easily shown that the above analysis remains valid for DA operation provided that we replace $A_{k}\left(r_{k} ; \hat{\theta}_{k}\right)$ with $\mathrm{a}_{\mathrm{k}}$, $\mathrm{p}\left(\mathrm{r}_{\mathrm{k}} ; \theta\right)$ with $\mathrm{p}\left(\mathrm{r}_{\mathrm{k}} \mid \mathrm{a}_{\mathrm{k}} ; \theta\right)$ and $\mathrm{CRB}_{\text {ind }}$ with $\mathrm{CRB}_{\mathrm{ps}}$.

\section{REFERENCES}

[1] A. D'Amico, A. D'Andrea and R.Reggiannini, "Efficient NonData-Aided Carrier and Clock Recovery for Sattelite DVB at Very Low Signal-to-Noise Ratio," IEEE J. on Sel. Areas in Commun., vol. 19, NO. 12, Dec. 2001 , pp. $2320-2330$

[2] L. Lu and G. Wilson, "Synchronization of Turbo Coded Modulation Systems at Low SNR," in Proc. Int. Conf. on Commun., Atlanta, GA, 1998, pp. 428-432

[3] L. Zhang and A. Burr, "Iterative Carrier Phase Recovery suited for Turbo-Coded systems," IEEE Trans. On Wireless Commun., vol. 3, NO. 6, Nov. 2004, pp. 2267-2276

[4] N. Noels, V. Lottici, A. Dejonghe, H. Steendam, M. Moeneclaey, M. Luise and L. Vandendorpe, "A Theoretical Framework for Soft Information Based Synchronization in Iterative (Turbo) Receivers," EURASIP J. on Wireless Commun. and Networking, vol. 2005, NO. 2, April 2005, pp. 117-129

[5] W. Oh and K. Cheun, "Joint decoding and carrier recovery algorithms for turbo codes," IEEE Commun. Lett., vol. 6, Sept. 2001, pp. 375377

[6] C. Langlais and M. Helard, "Phase carrier recovery for turbo codes over a sattelite link with the help of tentative deisions," in Proc. Intern. Symp. on Turbo Codes \& Relat. Topics, Brest, France, Sept. 2000, pp. 439-442

[7] A. Anastasopoulos and K.M.Chugg, " Adaptive iterative detection for phase tracking in turbo-coded systems," IEEE Trans. Commun., vol. 49, Dec. 2001, pp 2135-2144

[8] G. Colavolpe, A. Barbieri and G. Caire, "Iterative decoding in the presence of strong phase noise," submitted to IEEE J. on Sel. Areas in Commun., available at www. eurecom.fr $/ \sim$ caire

[9] N. Noels, H. Steendam and M. Moeneclaey, "A MaximumLikelihood Based FB Carrier Synchronizer for Turbo-Coded Systems", in Proc. IEEE Vehicular Technology Conference (VTC) spring 2005, Stockholm, Sweden, May 2005

[10] C. Georghiades and D. Snyder, "The Expectation-Maximization Algorithm for Symbol Unsynchronized Sequence Detection," IEEE Trans. On Commun., vol. 39, NO. 1, Jan. 1991, pp. 54-61

[11] R.A. Boyles, "On the convergence of the EM algorithm," J. Roy. Statist. Soc. B 45, No 1, pp. 47-50

[12] N. Noels, H. Steendam, M. Moeneclaey, "The Cramer-Rao Bound for Phase Estimation from Coded Linearly Modulated Signals," IEEE Commun. Lett., vol. 7, No. 5, May 2003, pp. 207-209

[13] H. L Van Trees, "Detection, Estimation, and Modulation Theory," New York, Wiley, 1990

[14] F. Kschinschang, B. Frey and H.-A. Loeliger, "Factor Graphs and the sum-product algorithm," IEEE Trans. Inform. Theory, vol. 47, NO. 2, Feb. 2001, pp. 498-519

[15] H. Meyr, M. Moeneclaey and S. A. Fechtel, "Digital Communication Receivers, Synchronization, Channel Estimation and Signal Processing," New York, Wiley, 1998

[16] L.R. Bahl, J. Cocke, F. Jelinek and J. Raviv, "Optimal decoding of linear codes for minimizing symbol error rate," IEEE Trans. Inform. Theory, vol. 20, March 1974, pp. 248-287

[17] W.G. Cowley, "Phase and Fequency estimation for PSK packets: bounds and algorithms," IEEE Trans. Comm., vol. 44, Jan. 1996, pp. 26-28 